Musées, Patrimoine et Culture scientifiques et techniques

$168 \mid 2016$

novembre-décembre 2016

\title{
Le transport des charges lourdes au musée départemental Arles antique
}

\section{Claude Sintes}

\section{OpenEdition \\ Journals}

Édition électronique

URL : http://journals.openedition.org/ocim/1716

DOI : $10.4000 /$ ocim. 1716

ISSN : 2108-646X

\section{Éditeur}

OCIM

Édition imprimée

Date de publication : 1 novembre 2016

Pagination : 21-24

ISSN : 0994-1908

Référence électronique

Claude Sintes, « Le transport des charges lourdes au musée départemental Arles antique », La Lettre de I'OCIM [En ligne], 168 | 2016, mis en ligne le 01 novembre 2017, consulté le 06 mai 2019. URL http://journals.openedition.org/ocim/1716 ; DOI : 10.4000/ocim.1716

Ce document a été généré automatiquement le 6 mai 2019.

Tous droits réservés 


\title{
Le transport des charges lourdes au musée départemental Arles antique
}

\author{
Claude Sintes
}

Le musée lapidaire de l'église Sainte-Anne, au début du XXe siècle.

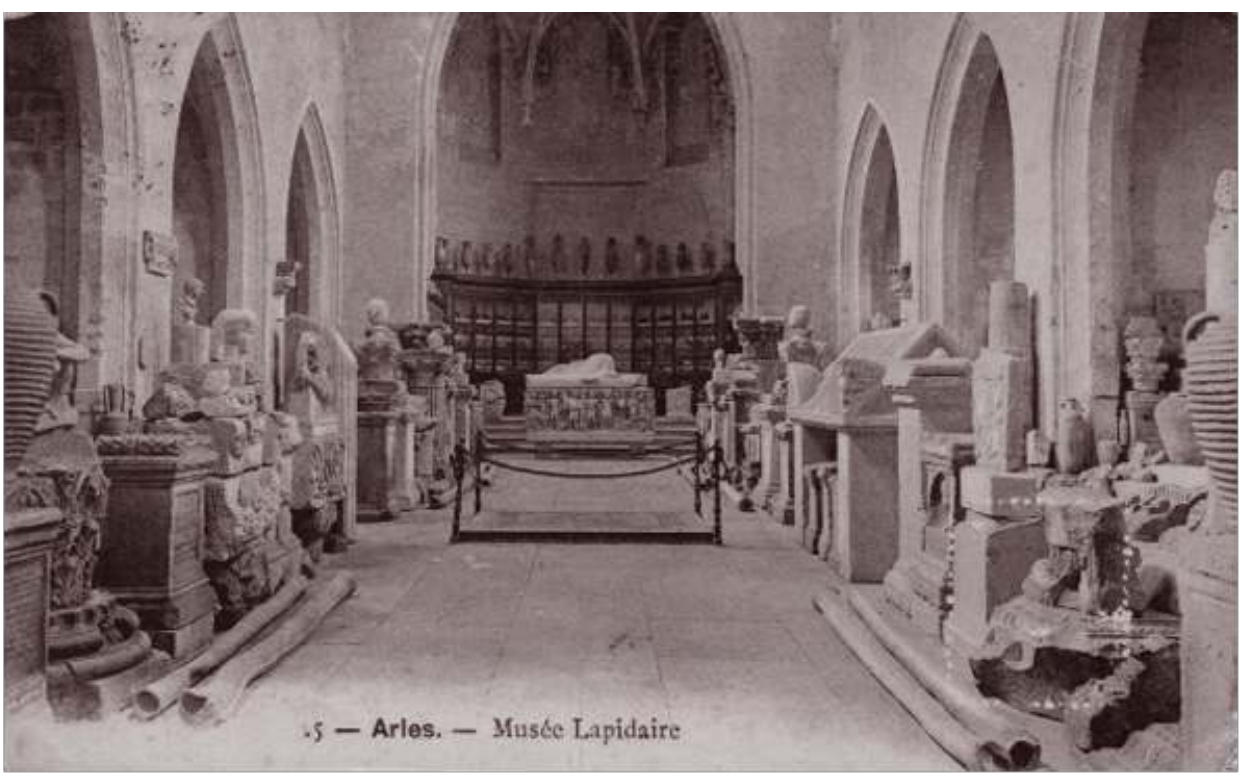

(c) DR

Pour ouvrir cette contribution par un truisme, il est possible de rappeler que la question du déplacement des collections ne se pose pas de la même manière dans un musée du timbre poste et dans un musée d'archéologie ! Certaines pièces y sont facilement manipulables comme la céramique ou la numismatique certes, mais très vite l'unité de mesure atteint le quintal avec les fragments d'architecture, la mosaïque ou la grande sculpture. À cela il faut ajouter l'encombrement de ces objets, leur fragilité malgré les apparences et l'inadéquation des ouvertures et des dégagements : quiconque a essayé de faire passer une borne miliaire fracturée, de deux tonnes, en biais par une porte trop 
petite, a pu le constater. Le tout naturellement dans les édifices les plus malcommodes qui soient, d'anciennes églises, temples ou hôtels particuliers dont le charme est aussi certain que l'abondance des volées d'escalier et des paliers trop étroits.

2 Cette situation, avec les risques de blessures pour le personnel que cela implique, les possibles dégradations des œuvres et le coût de chaque manipulation, prévalait à Arles, comme dans d'autres cités au prestigieux passé antique. Ainsi, à chaque nouvelle découverte archéologique volumineuse demandant une présentation au public, à chaque réaménagement d'espace, nos prédécesseurs ont dû mettre en place des chèvres, des tireforts et des platelages puis subir d'inévitables épaufrures ou, plus grave, quelques doigts écrasés. Or, les mouvements d'œuvres, n'ont pas manqué dans l'histoire de la collection arlésienne, ce qu'il convient de brièvement rappeler ici.

\section{Socles maçonnés du musée lapidaire après sa refonte par Jacques Latour.}

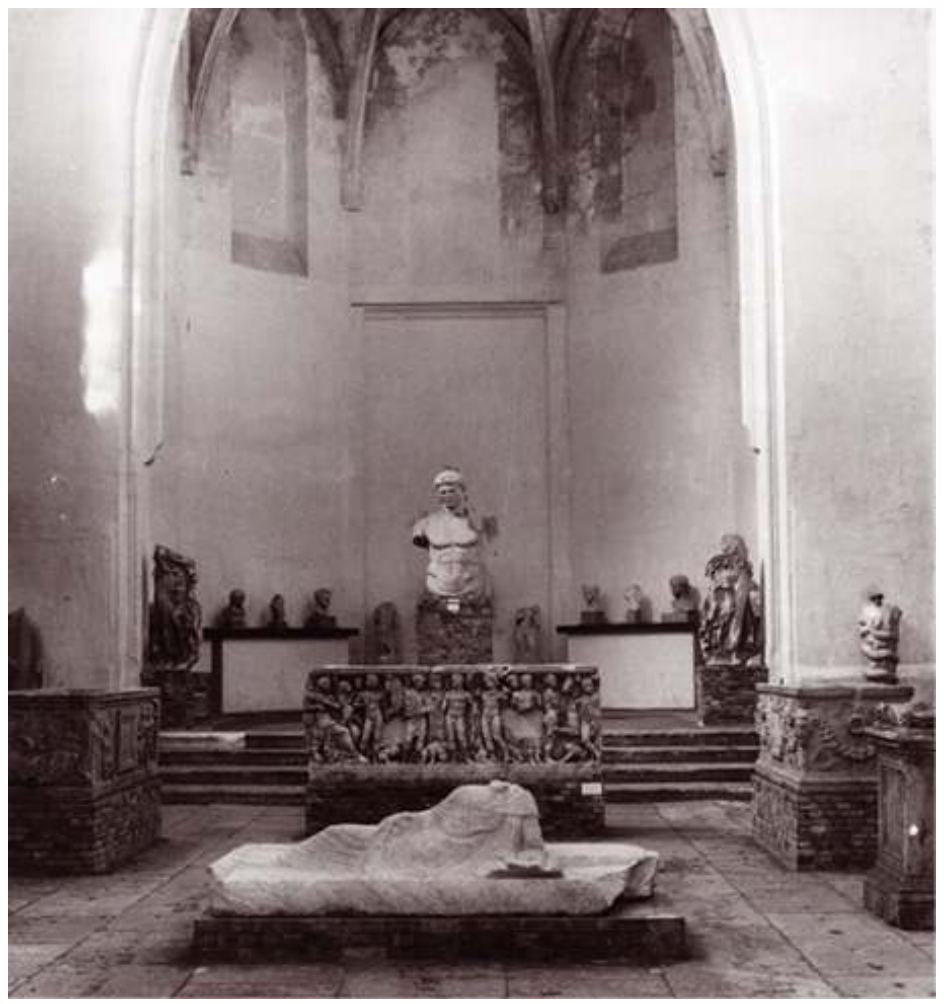

(c) Musées d'Arles/Michel Lacanaud

\section{Une collection sans domicile fixe}

Patiemment accumulées au fil des siècles par les érudits locaux, les autorités civiles ou ecclésiastiques, les collections archéologiques font partie de l'identité arlésienne depuis le XVI ${ }^{e}$ siècle. Avec la construction du musée de l'Arles et de la Provence antiques en 1995, ces œuvres sont présentées enfin dans un lieu digne de leur qualité mais, avant ce transfert, elles ont connu bien des vicissitudes.

Conservées à l'origine dans des endroits prestigieux mais peu adaptés comme le hall de l'Hôtel de Ville ou la cour du couvent de la Miséricorde, les pièces majeures vont être regroupées aux Alyscamps lors de la création du musée public d'Antiquités, en 1784. 
Comme le stipule un décret des Consuls d'Arles, pour la première fois il s'agissait de " rassembler et réunir dans un seul et même endroit les divers monuments d'antiquité qui se trouvaient épars dans la ville ou son terrain ... pour leur permettre d'être visités plus aisément par les curieux". Ce projet si novateur ne va pas pouvoir être conduit à son terme en raison des troubles de la Révolution. À la faveur de ces évènements, les collections et les tombeaux de la nécropole des Alyscamps sont vandalisés en partie (1794), pillés ou, pour certains, débités comme de la pierre à bâtir. Ajoutant à ces malheurs, l'État exige des "dons" de sarcophage ou de statues pour les musées de Marseille et pour le Louvre... La situation est telle que les pouvoirs publics envisagent un retour des œuvres les plus importantes dans le hall de l'Hôtel de Ville, faute de mieux.

5 La période qui suit, de 1805 à 1825, est assez confuse avec le ministère qui rappelle régulièrement son souhait d'envoyer des "monuments" à Paris et les responsables municipaux qui se montrent parfois peu efficaces dans la défense du patrimoine. Les collections vont être éparpillées puis rassemblées dans des lieux divers au gré des injonctions des uns et des réticences des autres. Le pire sera évité grâce à l'action de l'archéologue Pierre Véran (1744-1819) et du savant Aubin-Louis Millin (1859-1918) : les objets sont transférés une nouvelle fois depuis les Alyscamps jusqu'à l'église Sainte-Anne, de manière "provisoire". Ils vont y rester plus de cent ans. Pendant tout ce temps, le musée des Antiques est un dépôt lapidaire où voisinent en désordre sarcophages paléochrétiens, sculptures d'époque augustéenne ou copies en plâtre des chefs d'œuvres grecs. Au moins les déplacements incessants, si meurtriers pour du lapidaire fragile, ont été stoppés.

6 La première tentative rationnelle pour ordonner un discours scientifique intervient en 1925 lorsque le conservateur Fernand Benoit (1892-1969) décide de diviser les collections en "art païen" et "art chrétien", selon la terminologie de l'époque, ce qui va impliquer de nouveaux mouvements. L'ancienne chapelle du collège des Jésuites est mise à sa disposition pour abriter la prestigieuse série d'art paléochrétien. Elle ouvre au public en 1936 après une présentation des sarcophages et des stèles plus aérée que les entassements précédents. Après la Seconde Guerre mondiale, le conservateur Jacques Latour procède à une nouvelle refonte des musées en privilégiant cette fois l'esthétique. Il conçoit une scénographie agréable au prix d'une mise en réserve de la majorité des pièces : elles vont rester inaccessibles pendant près d'un demi siècle. Les témoins de l'époque racontent la difficulté pour ressortir les tombeaux et les fragments d'architecture de l'église, en construisant des platelages équipés de rails en bois suifé. Après transport, sur des camions à plateaux garnis de vieilles couvertures et de paille, il a fallu remonter cet imposant appareil quelques centaines de mètres plus loin afin d'introduire les mêmes éléments lapidaires par plans inclinés dans les caves du musée Réattu. 
Façade du musée départemental Arles antique

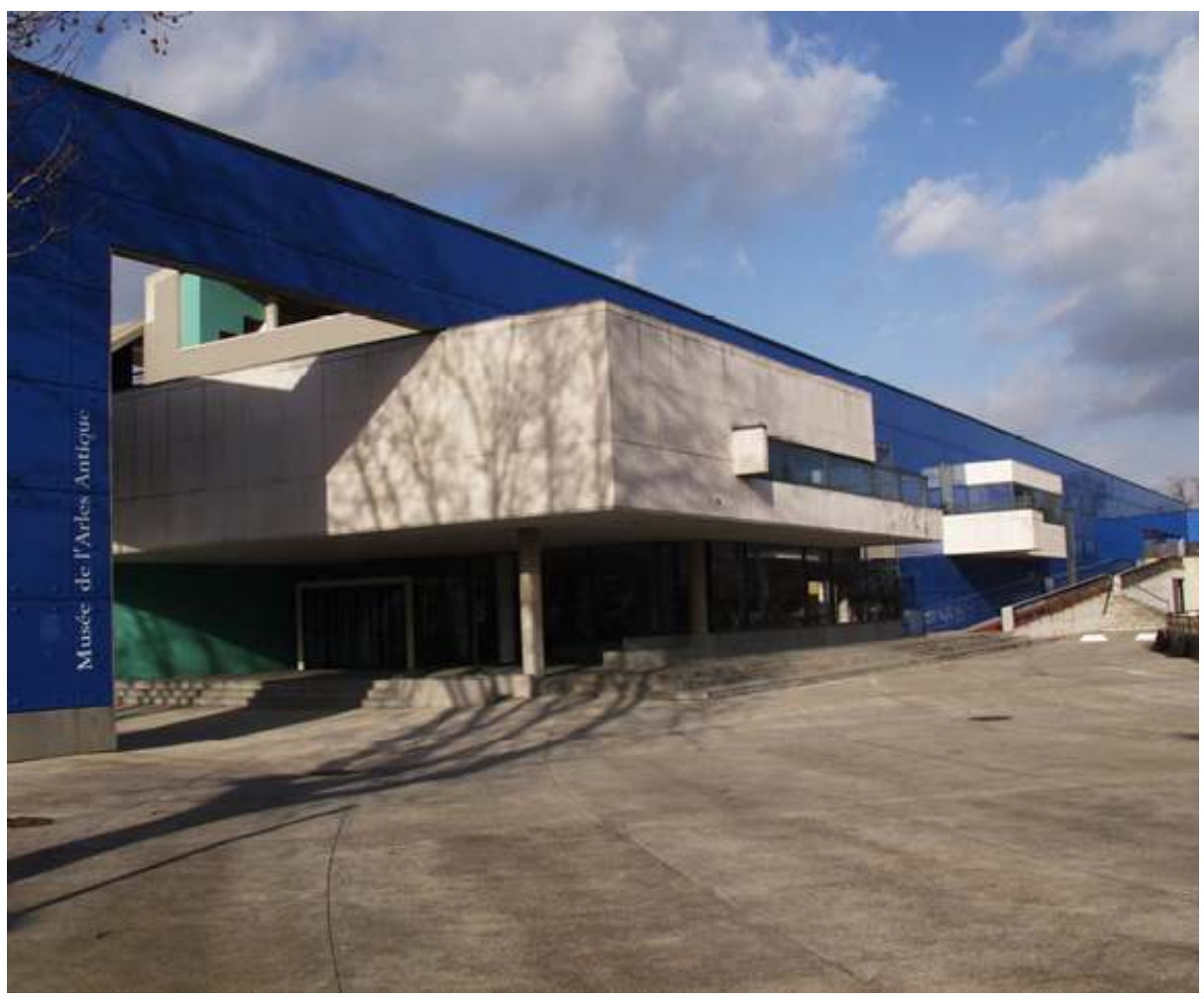

(c) Musées d'Arles/Michel Lacanaud

\section{Création d'un lieu adapté, le musée Arles antique}

7 C'est au conservateur Jean-Maurice Rouquette que revient l'idée d'un bâtiment regroupant les collections éparses dans les trois espaces (musée Païen, musée Chrétien, réserves du musée Réattu). Une fois le projet de création du "musée de l'Arles antique" validé au début des années 1980, il a surtout fallu éviter que les objets une fois en place n'en bougent plus ensuite pendant des décennies, interdisant les campagnes de restauration, les examens par les spécialistes et rendant périlleux le prêt à d'autres institutions. Le redéploiement des collections devait se faire selon un programme rigoureux, dans un espace de présentation suffisant et avec des cheminements et des moyens de levage adaptés. L'édifice allait en outre abriter des réserves, des laboratoires dédiés à la recherche archéologique, à la restauration des mosaïques et des salles de travaux pratiques pour l'enseignement supérieur. Le lien entre ces coulisses et la salle de présentation devait permettre le transport des collections les plus lourdes sans mobiliser des moyens dispendieux ou des mises en place compliquées.

Un concours organisé en 1983 retenait l'architecte Henri Ciriani, dont le projet à l'esthétique très contemporaine répondait au mieux aux différents termes du programme. Instruit par les difficultés de nos prédécesseurs, c'est avec une expérience "multi séculaire" des grosses charges que Jean-Maurice Rouquette a pu écrire le programme et associer l'architecte à cette préoccupation. Anticiper le déplacement des pièces lourdes en dessinant un bâtiment adapté peut paraitre aujourd'hui du simple bon sens mais, au début des années 1980, ce n'était pas encore si évident que cela. Pour 
l'anecdote, on peut signaler que l'un des projets du concours d'architecture prévoyait de présenter la collection de sarcophages à l'étage, dans un encorbellement au dessus du fleuve, et en les y installant au moyen d'une grue.

\section{La circulation dans les coulisses}

Dès son origine, le projet scientifique du musée visait à maitriser la chaîne opératoire, depuis la fouille sur le terrain jusqu'à la mise à disposition publique des collections restaurées. Cette fluidité revendiquée imposait la plus grande souplesse pour la manutention et le déplacement de n'importe quelle charge. Le principe architectural directeur a donc été de concevoir un bâtiment de plain-pied, sans aucune rupture par emmarchement ou plateau entre les diverses zones d'activités et la salle de présentation. Les accès sont facilités par un quai de déchargement au dessus duquel est installé un pont roulant équipé d'un treuil électrique. Dans le financement du projet un budget d'investissement a été consacré à l'achat d'un chariot élévateur d'une capacité de cinq tonnes et les circulations dessinées en tenant compte de son encombrement.

Installation dans les collections d'une mosaïque restaurée dans l'atelier du musée

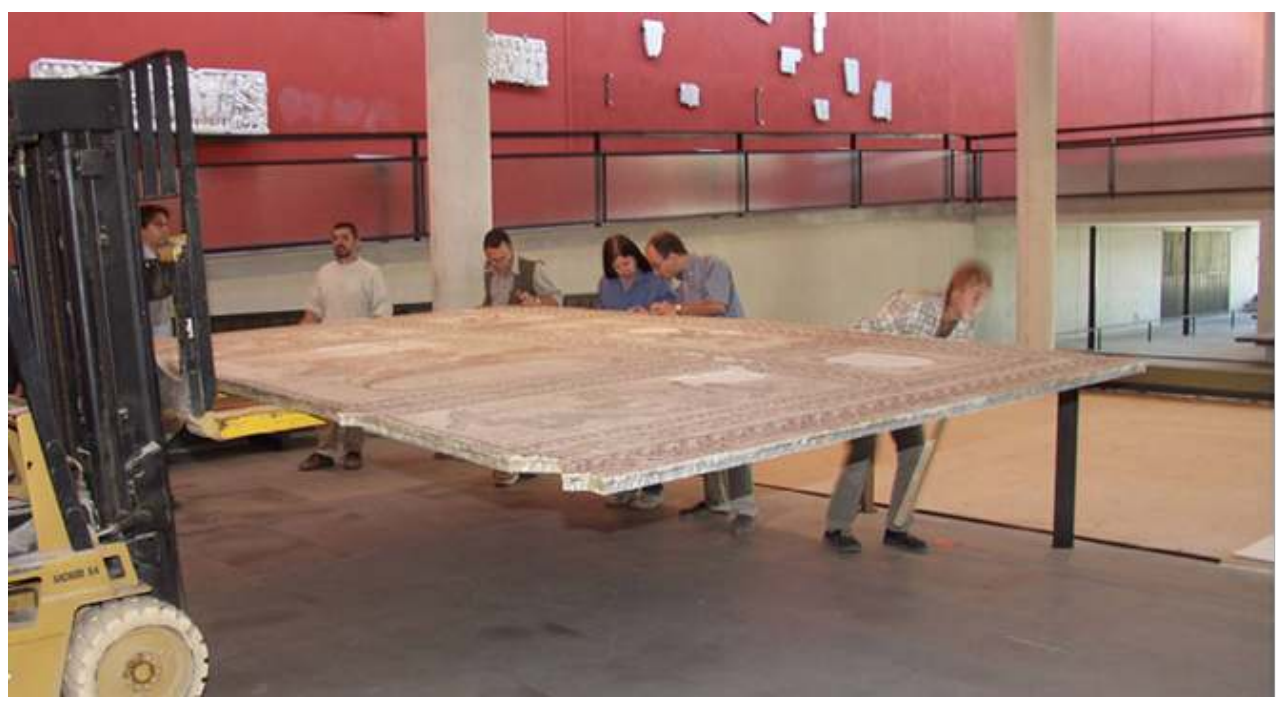

(c) Musées d'Arles/Michel Lacanaud

10 C'est dans les coulisses que l'on comprend au mieux ce souci de maitrise globale que l'on pourrait presque qualifier d'industriel et qui a présidé à la conception de l'établissement. L'aile sud est ainsi consacrée à l'activité scientifique du musée : elle abrite l'équipe de fouilles, les ateliers de restauration, les réserves et les différents équipements d'étude des collections. Desservie par une entrée indépendante, elle accueille les objets découverts sur le terrain. Dès leur arrivée toutes ces pièces, quels que soient leur poids, sont palettisées et acheminées par des engins de manutention. L'accueil des objets se fait dans une unité de soins où ils passent par une salle de lavage spécialisée, puis dans une salle de séchage, avant d'atteindre la salle d'examen où ils sont immatriculés, identifiés et triés selon un premier diagnostic des archéologues. Ils sont alors dirigés vers la salle de prises de vues photographiques afin de compléter leur fiche d'enregistrement. La destination finale dépend de leur état de conservation. Si celui-ci est satisfaisant, ils gagnent par la rue intérieure de l'aile scientifique un espace de présentation - permanente ou 
temporaire -, les différents laboratoires de recherche ou les salles de réserves. Une salle de consultation contiguë permet de recevoir les chercheurs et de préparer les publications.

Un des sarcophages du musée sur son socle muni d'une plaque métallique de protection

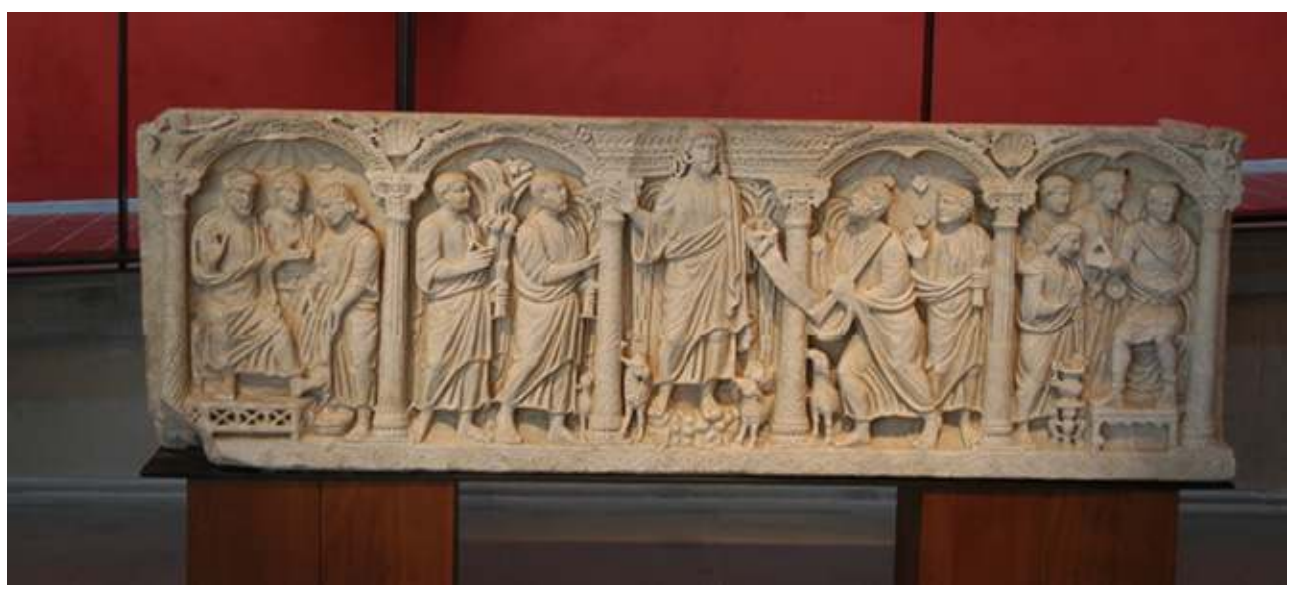

(c) Musée départemental Arles Antique/C. Sintes

Cet ensemble a été conçu pour assurer le traitement des collections du fonds et les accroissements prévisibles, compte tenu du rythme des travaux sur le terrain. Lorsque l'état sanitaire des objets d'acquisition récente ou de ceux qui sont déjà intégrés dans les collections nécessite un traitement préventif ou une restauration, ils sont dirigés vers l'un des laboratoires spécialisés pour la céramique, les enduits peints ou la mosaïque. Un effort tout particulier a été déployé pour la mise en œuvre de l'atelier de restauration des mosaïques en raison de l'importance de la collection arlésienne et du grand nombre de pavements que mettent au jour nos chantiers de fouilles. Un équipement de plus de $400 \mathrm{~m}$ ${ }^{2}$ comprenant une cour de sablage et une vaste salle de montage, susceptible de permettre les interventions les plus complexes, a été confié aux restaurateurs. Mosaïques et opus sectile sont pris en charge dès leur découverte sur le terrain, déposés, remontés sur un nouveau support, restaurés avec le plus parfait respect des éléments anciens et exposés à l'attention du public. Comme indiqué plus haut les cheminements existant entre ces différents lieux de recherche, de stockage ou de présentation ont été dessinés en tenant compte des dimensions maximales manipulables en intérieur : surface et longueur des mosaïques, poids des pièces les plus massives, encombrement des œuvres sculptées. Les largeurs de portes, les renforcements de sol pour charges roulantes et les dégagements nécessaires dans tout le musée découlent de ces mesures étalons.

\section{La circulation dans les réserves et dans la collection permanente}

Le musée ne peut présenter l'ensemble des collections qu'il conserve (estimé à plus de 15 000 objets), faute de place et parce qu'il comporte une bonne part de collections d'étude. L'essentiel de ces pièces est actuellement réparti dans deux réserves $\left(720 \mathrm{~m}^{2}\right.$ en tout), l'une dévolue au lapidaire et l'autre pour tous les objets de petite dimension, conditionnés par types de matériaux. Cette dernière se situe à l'étage mais est accessibles grâce à un monte-charge acceptant les engins de manutention légers comme les transpalettes. La 
réserve lapidaire lourde possède des étagères de béton ou d'acier pouvant supporter des charges de plusieurs tonnes, toutes accessible par engin de levage. Les sarcophages et stèles non présentés y sont stockés sur plusieurs rangs mais peuvent être sortis à la demande lors d'expositions temporaires, d'examens par des chercheurs ou des restaurateurs. Le lien avec la salle d'exposition a été soigneusement défini : une porte coulissante blindée assure le passage de plain-pied depuis la réserve vers les salles permanentes ou la salle temporaire et la scénographie fixe a été installée de manière à ménager un passage suffisant au long de l'itinéraire. Tout point du musée est dés lors atteignable par les engins de manutention, ce qui permet une souplesse bienvenue pour les reprises de présentation ou pour les mouvements lors des prêts.

Ce principe a été appliqué à l'ensemble des salles de présentation. Outre le cheminement calculé pour un engin en charge, ce dont on a parlé, la scénographie a tenu compte des contraintes de manutention. Ainsi, dès l'origine, les socles des pièces lourdes ont été dessinés afin de garantir une prise facile associée à un débattement en hauteur convenable. On est loin des socles maçonnés et massifs des vieux musées d'art païen ou chrétien, n'autorisant plus aucun déplacement une fois l'œuvre installée. Les sarcophages du musée d'Arles sont ainsi posés sur des cubes de bois espacés acceptant le passage des fourches de l'élévateur et leur réglage en largeur. La facilité de ce système comporte cependant le risque de voir les collections se détériorer. Pour y remédier, il a été décidé de poser chaque tombeau sur une plaque métallique épaisse, assurant le maintien de l'œuvre lors des déplacements et évitant les éraflures ou écrasement de matière au moment de la mise en place des fourches de l'engin malgré les précautions prises.

En conclusion, et pour donner des exemples de ce qu'un musée préparé au transport des charges lourdes permet, on peut évoquer l'exposition Ingres et l'antique (2 octobre 2006-2 janvier 2007) où la "Minerve Ingres" du Louvre a été acheminée dans nos salles sans encombre malgré ses quatre tonnes et demi de marbre fragile, ou bien l'exposition temporaire Rodin et l'antique (6 avril-1 ${ }^{\mathrm{er}}$ septembre 2013). Lors de cette manifestation on a présenté l'une des sculptures les plus volumineuses jamais accueillies dans nos murs, le Monument à Victor Hugo du Palais-Royal, bronze de près de trois mètres de large et d'un mètre quatre vingt dix de haut inaugurée en 1909. Pour décharger la caisse sur notre quai grâce à l'engin élévateur, préparer l'œuvre, la transférer en réserve puis dans les salles grâce à deux transpalettes, la mettre sur socle enfin, quelques hommes et une demi journée ont suffi : je n'ose imaginer combien de jours et de peine il eut fallu à nos prédécesseurs dans le vieux musée d'art chrétien.

\section{RÉSUMÉS}

Après avoir rappelé l'histoire et la genèse du musée départemental Arles antique en évoquant notamment les difficultés rencontrées pour le déplacement des œuvres encombrantes et lourdes, l'auteur montre comment, lors du récent réaménagement du musée, les contraintes liées à la manutention et à la circulation de ces collections spécifiques ont nécessité une adaptation de la scénographie. 
INDEX

Mots-clés : transport, collection

\section{AUTEUR}

CLAUDE SINTES

conservateur en chef du patrimoine, directeur du musée départemental Arles antique claude.sintes@cg13.fr 